\title{
The Dynamical and Structural Basis of Brain Activity
}

\author{
Gustavo Deco, Viktor Jirsa, and Karl J. Friston
}

Summary

Global network dynamics over distributed brain areas emerge from the local dynamics of each brain area. Conversely, global dynamics constrain local activity such that the whole system becomes self-organizing. The implicit coupling between local and global scales induces a form of circular causality that is characteristic of complex, coupled systems that show self-organization, such as the brain. Here we present a network model based on spiking neurons at the local level and large-scale anatomic connectivity matrices at the global level. We demonstrate that this multiscale network displays endogenous or autonomous dynamics of the sort observed in resting-state studies. Our special focus here is on the genesis of itinerant (wandering) dynamics and the role of multistable attractors, which are involved in the generation of empirically known functional connectivity patterns, if the global coupling causes the dynamics to operate in the critical regime. Our results provide once again support for the hypothesis that endogenous brain activity is critical.

\subsection{Introduction}

Since the inception of experimental brain research, one of the most important objectives of neuroscience has been to understand the neuronal and cortical mechanisms underlying perceptual and cognitive functions. Early approaches to this challenging question originated one of the first influential brain theories, namely localizationism, which postulates that the brain is functionally segregated (i.e., that parts of the brain, and not the whole, perform specific functions). Localizationism was motivated by Franz Joseph Gall's theory of phrenology (Gall \& Spurzheim, 1809). Although phrenology was not based on rigorous experimental facts, empirical support came from numerous experiments during the 19th century. Anatomists and physiologists such as Pierre Broca, Carl Wernicke, and many others were able to identify specific regions in 
animals and humans associated with particular brain functions, so that the concept of functional segregation was firmly established by the end of the nineteenth century (for a review, see Feinberg \& Farah, 1997). Localizationism enjoyed a renaissance with the advent of functional brain imaging (e.g., functional magnetic resonance imaging; fMRI), which confirmed that specific functions, elicited experimentally, activated particular regions of the brain. However, localizationism is no longer the dominant paradigm in cognitive neuroscience: Many fMRI (and human electroencephalography (EEG) and magnetoencephalography (MEG) as well as animal cell recording) studies support the view that neuronal computations are distributed and engage a network of distributed brain areas (e.g., Cabeza \& Nyberg, 2000). This is known as functional integration. This perspective, originally proposed by Flourens (1824) as a response to localizationism, is not inconsistent with functional segregation but emphasizes the interactions among functionally segregated brain areas. Nowadays, there is overwhelming evidence that all representations in the brain are distributed. Perceptions, memories, and even emotions are represented in a distributed manner; hence, a deeper understanding of the mechanisms underlying distributed processing is a central question for neuroscience. The main tools for characterizing distributed brain processing to date include the estimation of functional and effective connectivity from brain-imaging time series (McIntosh \& Gonzalez-Lima, 1994; Cordes et al., 2000; Friston, Harrison, \& Penny, 2003). Functional connectivity is defined as the statistical dependence between remote neurophysiologic events and is usually assessed with simple correlation or coherence analyses of fMRI or electrophysiologic time series. Conversely, effective connectivity is defined as the influence one system exerts over another and rests explicitly on an underlying model of neuronal dynamics (Friston et al., 2000). These dynamics are fundamental for processing of information at both a local level and a global level. They reconcile the apparently conflicting views of local versus global representation. Global network dynamics over distributed brain areas emerge from the local dynamics of each brain area. Conversely, global dynamics constrain local activity such that the whole system becomes self-organizing. The implicit coupling between local and global scales induces a form of circular causality that is characteristic of complex, coupled systems that show self-organization, like the brain. An important example of this is the slaving principle, where microscopic modes or patterns of activity become enslaved by a small number of macroscopic modes. In this chapter, we will take a look at how the dynamics of neuronal popula- 
tions within a cortical area are enslaved by large-scale intercortical dynamics.

This chapter comprises two sections. In the first, we will consider models of brain dynamics from both local and global perspectives. We will present a specific model based on spiking neurons at the local level and large-scale anatomic connectivity matrices at the global level. This model is used in the second section to provide a quantitative example of how to model dynamical mechanisms in the brain. The second section focuses on endogenous or autonomous dynamics of the sort observed in resting-state studies. Our special focus here is on the genesis of itinerant (wandering) dynamics and the role of multistable attractors. In brief, we will show that the model described in the first section predicts empirical correlations in the brain when, and only when, global coupling brings it into a critical regime. We conclude with a brief discussion of the importance of autonomous dynamics in neuroscience and the universal properties that they might possess.

\subsection{Attractors and Brain Dynamics}

Computational neuroscience tries to describe the dynamics of networks of neurons and synapses with realistic models to reproduce emergent properties or predict observed neurophysiology (single- and multiple-cell recordings, local field potentials, optical imaging, EEG, MEG, fMRI) and associated behavior. Recently, a powerful theoretical framework known as attractor theory (see, e.g., Brunel \& Wang, 2001) was introduced to capture the neural computations inherent in cognitive functions like attention, memory, and decision making. This theoretical framework is based on mathematical models formulated at the level of neuronal spiking and synaptic activity. Analysis of networks of neurons modeled at the integrate-and-fire level enables the study of many aspects of brain function: from the spiking activity of single neurons and the effects of pharmacological agents on synaptic currents, through to fMRI and neuropsychological findings. In the following, we describe the integration of local and global cortical dynamics in the setting of attractor networks.

\subsubsection{A Local Model: Spiking Attractor Networks}

To model a local brain area (i.e., a node in the global brain network), we will use a biophysically realistic attractor network model consisting of identical integrate-and-fire spiking neurons with synaptic dynamics 
(Brunel \& Wang, 2001). Extensions to ensembles of nonidentical neurons have been developed for electric (Assisi, Jirsa, \& Kelso, 2005; Jirsa, 2007; Stefanescu \& Jirsa, 2008) and synaptic (Jirsa \& Stefanescu, 2010; Stefanescu \& Jirsa, 2011) coupling. Attractor networks of spiking neurons are dynamical systems that have the tendency to settle in stationary states or fixed points called "attractors," typically characterized by a stable pattern of firing. External or even intrinsic noise that manifests in the form of finite size effects can destabilize these fixed-point attractors, inducing transitions among different attractors. The dynamics of these networks can be described by dynamical equations describing each neuron and how they influence each other through their coupling or effective connectivity. Spikes arriving at a given synapse provide an input to the neuron, which induces postsynaptic excitatory or inhibitory potentials. These are described by a low-pass filtering mediated by postsynaptic receptors. For example, the total synaptic current may be assumed to be the sum of glutamatergic (AMPA and NMDA) and GABAergic recurrent inhibitory currents. The parameters describing the biophysics of the neurons and synapses (conductances, latencies, etc.) are generally selected from a biologically realistic range. Each local attractor network typically contains a large number of excitatory pyramidal neurons and inhibitory neurons $(100-10,000)$. We will use local attractor networks whose neurons are organized into two sets of populations (see figure 1.1); namely, an inhibitory population and an excitatory population. The key free parameters controlling the dynamics of the network are the strength of the recurrent excitatory and inhibitory synaptic connections. All neurons in a local network also receive an external background input modeled by uncorrelated Poisson spike trains, whose time-varying rate is given by a noisy Ornstein-Uhlenbeck process representing noisy fluctuations. External sensory or task-dependent inputs can be simulated in the same way, using higher mean input rates.

In what follows, we summarize the mathematical description of spiking and synaptic dynamics. The particular model that we use here is fairly typical of (neural mass) models specified to this level of detail. There are many other forms of model; for example, those based on multiple compartments for each neuron. However, the basic form of the differential equations and the state variables they describe are usually quite similar.

The integrate-and-fire (IF) model of a neuron is specified by the dynamics of its membrane potential $V(t)$. An IF neuron can be described by a basic RC-circuit consisting of a cell membrane capacitance $C_{m}$ and a membrane resistance $R_{m}$. If the membrane potential is below a given 


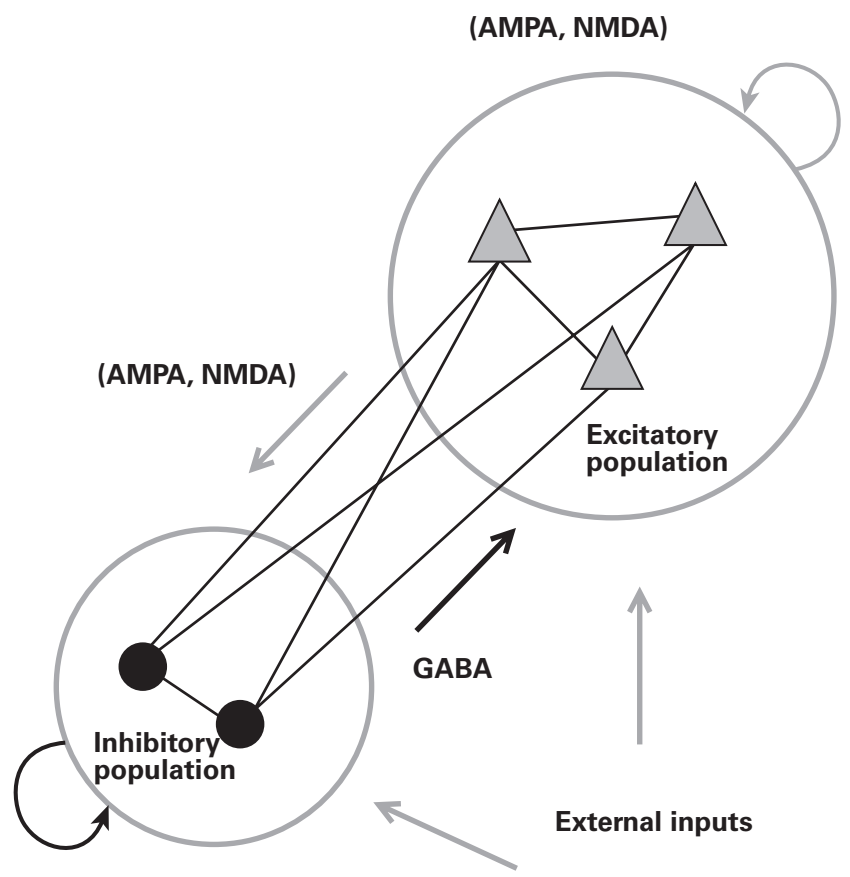

GABA

Figure 1.1

A local network. The network consists of spiking neurons with realistic AMPA, NMDA, and GABA synaptic dynamics. The network contains excitatory pyramidal cells and inhibitory interneurons.

threshold $V_{t h r}$ (subthreshold dynamics), then the membrane potential of each neuron in the network is given by the equation

$C_{m} \frac{d V(t)}{d t}=-g_{m}\left[V(t)-V_{L}\right]-I_{\text {syn }}(t)$,

where $g_{m}=1 / R_{m}$ is the membrane leak conductance, $V_{L}$ is the resting potential, and $I_{s y n}$ is the synaptic current. The membrane time constant is defined by $\tau_{m}=C_{m} / g_{m}$. When the voltage across the membrane reaches threshold, the neuron generates a spike, which is then transmitted to other neurons, and the membrane potential is instantaneously reset to $V_{\text {reset }}$ and maintained there for a refractory period $\tau_{\text {ref }}$, during which time the neuron is unable to produce further spikes.

Input currents from connected neurons or from external inputs drive the membrane potential: The spikes arriving at a synapse induce post- 
synaptic excitatory or inhibitory potentials. The total synaptic current is given by the sum of glutamatergic AMPA $\left(I_{A M P A, e x t}\right)$ mediated external excitatory currents, AMPA $\left(I_{A M P A, r e c}\right)$ and NMDA $\left(I_{N M D A, r e c}\right)$ mediated recurrent excitatory currents, and GABAergic recurrent inhibitory currents $\left(I_{G A B A}\right)$ :

$I_{\mathrm{syn}}=I_{\mathrm{AMPA}, \mathrm{ext}}+I_{\mathrm{AMPA}, \mathrm{rec}}+I_{\mathrm{NMDA}, \mathrm{rec}}+I_{\mathrm{GABA}}$,

where

$$
\begin{aligned}
& I_{A M P A, e x t}(t)=g_{A M P A, \text { ext }}\left[V(t)-V_{E}\right] \sum_{j=1}^{N_{\text {ext }}} s_{j}^{A M P A, e x t}(t) \\
& I_{A M P A, \text { rec }}(t)=g_{A M P A, r e c}\left[V(t)-V_{E}\right] \sum_{j=1}^{N_{E}} w_{j} s_{j}^{A M P A, r e c}(t) \\
& I_{N M D A, r e c}(t)=\frac{g_{N M D A, r e c}\left[V(t)-V_{E}\right]}{1+\gamma e^{-\beta V(t)}} \sum_{j=1}^{N_{E}} w_{j} s_{j}^{N M D A, r e c}(t) \\
& I_{\mathrm{GABA}}(t)=g_{\mathrm{GABA}}\left[V(t)-V_{I}\right] \sum_{j=1}^{N_{I}} w_{j} s_{j}^{\mathrm{GABA}}(t) .
\end{aligned}
$$

Here, $g_{A M P A, \text { ext }}, g_{A M P A, r e c}, g_{N M D A, r e c}$, and $g_{G A B A}$ are the synaptic conductances, and $V_{E}$ and $V_{I}$ are the excitatory and inhibitory reversal potentials, respectively. The dimensionless parameters $w_{j}$ of the connections are the synaptic weights. The NMDA currents are voltage dependent and are modulated by intracellular magnesium concentration. The gating variables $s_{j}^{i}(t)$ are the fractions of open channels of neurons and are given by

$$
\begin{aligned}
& \frac{d s_{j}^{A M P A, \text { ext }}(t)}{d t}=-\frac{s_{j}^{A M P A, \text { ext }}(t)}{\tau_{A M P A}}+\sum_{k} \delta\left(t-t_{j}^{k}\right) \\
& \frac{d s_{j}^{A M P A, \text { rec }}(t)}{d t}=-\frac{s_{j}^{A M P A, \text { rec }}(t)}{\tau_{A M P A}}+\sum_{k} \delta\left(t-t_{j}^{k}\right) \\
& \frac{d s_{j}^{N M D A, \text { rec }}(t)}{d t}=-\frac{s_{j}^{N M D A, \text { rec }}(t)}{\tau_{N M D A, \text { decay }}}+\alpha x_{j}(t)\left(1-s_{j}^{N M D A, \text { rec }}(t)\right) \\
& \frac{d x_{j}^{N M D A, \text { rec }}(t)}{d t}=-\frac{x_{j}^{N M D A, \text { rec }}(t)}{\tau_{A M P A, r i s e}}+\sum_{k} \delta\left(t-t_{j}^{k}\right)
\end{aligned}
$$


$\frac{d s_{j}^{G A B A}(t)}{d t}=-\frac{s_{j}^{G A B A}(t)}{\tau_{G A B A}}+\sum_{k} \delta\left(t-t_{j}^{k}\right)$.

The sums over the index $k$ represent all the spikes emitted by the presynaptic neuron $j$ (at times $t_{j}^{k}$ ). In the equations above, $\tau_{N M D A, \text { rise }}$ and $\tau_{N M D A \text {, decay }}$ are the rise and decays times for the NMDA synapses, and $\tau_{A M P A}$ and $\tau_{G A B A}$ are the decay times for AMPA and GABA synapses. The rise times of both AMPA and GABA synaptic currents are neglected because they are short $(<1 \mathrm{~ms})$. This concludes our description of the local model in terms of differential equations that describe the dynamics of each neuron in the populations considered. We will now turn our attention to the model of macroscopic or global dynamics.

\subsubsection{A Global Model: The Connectome}

The global brain model considered here is a network of local attractor networks described earlier. Crucially, the between-area (extrinsic) connections between different cortical areas are specified by a neuroanatomic matrix. We assume that the extrinsic connections between two distinct brain areas describe the density of synaptic connections between neurons in those areas. We weight those inter-areal connections by a coupling strength specified in the neuroanatomic matrix (numbers of fibers connecting those regions) and a global factor that plays the role of a control parameter. This control parameter will be used to study the dynamics and fixed points of the global system. Because the global system is defined in terms of its extrinsic connectivity, we can model global dynamics using connectivity data from macaque or humans. In the macaque case, we use the neuroanatomic matrix from the CoCoMac database (Kötter \& Wanke, 2005), which describes the connectivity among approximately 40 cortical areas in one hemisphere of the macaque brain. Figure 1.2B shows an example of such a matrix of connection strengths. The center coordinates of each cortical area can be obtained from the geometry defined in the Automatic Anatomic Labeling cortical surface template of a human hemisphere (Kötter \& Wanke, 2005). The length of the extrinsic connections (and consequently the delay in the transmission of spikes) can be evaluated from the distance between target and source regions. In the human case, we can use neuroanatomic information obtained by diffusion weighted tensor imaging (DTI) and diffusion spectrum imaging (DSI) tractography. An example is the neuroanatomic matrix of Hagmann et al. (2008: Briefly, after diffusion spec- 

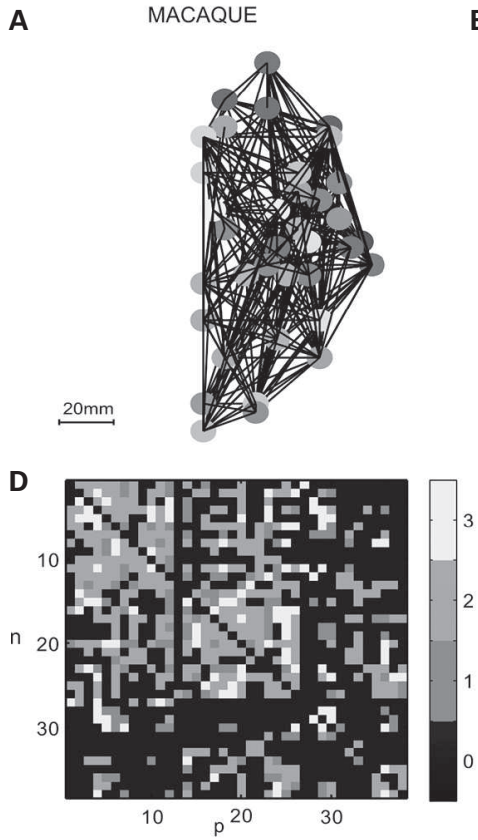

B

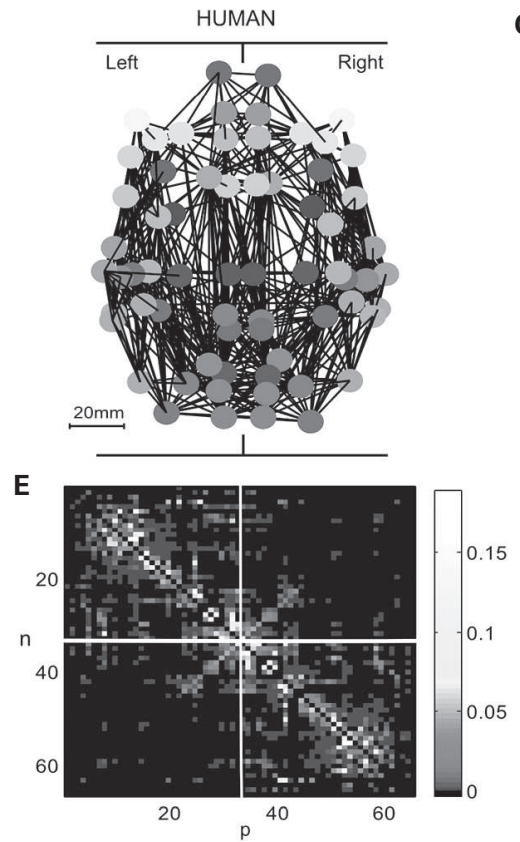

C

\begin{tabular}{|c|c|c|c|c|}
\hline & & & Huma & \\
\hline Mac & que & & right & left \\
\hline & & ENT & 1 & 66 \\
\hline A2 & 1 & PARH & 2 & 65 \\
\hline $\begin{array}{l}\text { PFCDL } \\
\text { PFCM }\end{array}$ & $\begin{array}{l}2 \\
3\end{array}$ & TP & 3 & 64 \\
\hline $\begin{array}{l}\text { PFCM } \\
\text { PFCORB }\end{array}$ & $\begin{array}{l}3 \\
4\end{array}$ & FP & 4 & 63 \\
\hline PFCPOL & 5 & FUS & 5 & 62 \\
\hline PFCVL & 6 & $\mathrm{TT}$ & 6 & 61 \\
\hline PHC & 7 & LOCC & 7 & 60 \\
\hline TCC & 8 & $\mathrm{SP}$ & 8 & 59 \\
\hline $\begin{array}{l}\text { TCl } \\
\text { TCPOL }\end{array}$ & $\begin{array}{c}9 \\
10\end{array}$ & IT & 9 & 58 \\
\hline TCS & 11 & IP & 10 & 57 \\
\hline TCV & 12 & SMAR & 11 & 56 \\
\hline Pulvinar & 13 & BSTS & 12 & 55 \\
\hline $\begin{array}{l}\text { CCP } \\
\text { CCR }\end{array}$ & $\begin{array}{l}14 \\
15\end{array}$ & MT & 13 & 54 \\
\hline FEF & $\begin{array}{l}15 \\
16\end{array}$ & ST & 14 & 53 \\
\hline PCIP & 17 & PSTC & 15 & 52 \\
\hline PCM & 18 & PREC & 16 & 51 \\
\hline PCS & 19 & $\mathrm{CMF}$ & 17 & 50 \\
\hline $\begin{array}{l}\text { PFCCL } \\
\text { V2 }\end{array}$ & $\begin{array}{l}20 \\
21\end{array}$ & POPE & 18 & 49 \\
\hline $\begin{array}{l}\text { V2 } \\
\text { VACV }\end{array}$ & $\begin{array}{l}21 \\
22\end{array}$ & PTRI & 19 & 48 \\
\hline $\mathrm{CCA}$ & 23 & RMF & 20 & 47 \\
\hline $\mathrm{PCl}$ & 24 & PORB & 21 & 46 \\
\hline PMCDL & 25 & LOF & 22 & 45 \\
\hline PMCM & 26 & CAC & 23 & 44 \\
\hline $\begin{array}{l}\text { A1 } \\
\text { Ccs }\end{array}$ & $\begin{array}{l}27 \\
28\end{array}$ & RAC & 24 & 43 \\
\hline IA & 29 & SF & 25 & 42 \\
\hline IP & 30 & MOF & 26 & 41 \\
\hline PFCDM & 31 & LING & 27 & 40 \\
\hline $\begin{array}{ll}V 1 \\
V A c\end{array}$ & 32 & PCAL & 28 & 39 \\
\hline $\begin{array}{l}\text { VACD } \\
\text { ThalAM }\end{array}$ & $\begin{array}{l}33 \\
34\end{array}$ & CUN & 29 & 38 \\
\hline M1 & 35 & PARC & 30 & 37 \\
\hline PMCVL & 36 & ISTC & 31 & 36 \\
\hline s1 & 37 & PCUN & 32 & 35 \\
\hline S2 & 38 & PC & 33 & 34 \\
\hline
\end{tabular}

Figure 1.2 (plate 1)

Structural connectivity of the macaque and the human cortex. (A) Three-dimensional representation of the CoCoMac (left) and the human connectome (right) networks (view from above). The nodes representing anatomic regions are placed at their central coordinates. (B) Connection strength matrices for the CoCoMac (left) and the $66 \times 66$ human connectome (right) where $n$ is the source region and $p$ is the target. The connectome is ordered in such a way that corresponding contralateral regions are arranged symmetrically, with respect to the matrix center; the anti-diagonal reveals the existing connections between these contralateral regions. The white lines separate the two hemispheres. The cortical regions for the CoCoMac and for the human connectome are shown in the left and right subpanels and index the regions in (A).

trum and T1-weighted MRI acquisitions, the segmented gray matter was partitioned into approximately 1000 anatomic regions of interest (ROIs). White matter tractography identified voxel pairs that were connected to specify a neuroanatomic matrix. Note that tractography does not give the directionality of the fibers mediating the connection. This means that the connectivity matrix is symmetric, which may have consequences for the observable network dynamics (Knock et al., 2009; Jirsa, Sporns, Breakspear, Deco, \& McIntosh, 2010), but will remain within limits unless the symmetry breaking is large. The resulting structural connectivity (SC) matrices were then averaged across five subjects. To downsample the SC to 66 regions, the connection strength between two regions was calculated by summing all incoming connection strengths to the 
target region and dividing by its number of ROIs. Clearly, because the number of ROIs depends on the region, the downsampled connectivity matrix is no longer symmetric. As the local dynamical model already models within-area (intrinsic) connectivity, the connection of a region to itself is generally omitted from extrinsic connectivity matrices (see figure 1.2, plate 1). The length of a connection between two regions can be calculated as the average length across all connecting tractography fibers.

In the next section, we will simulate brain responses that arise from spontaneous fluctuations in this dynamical model. At present, most neuroimaging studies of distributed activity of this sort are performed with fMRI. We therefore need to model the mapping between the neuronal dynamics, specified in terms of depolarization, to the sorts of signals observed with fMRI. These are referred to as blood oxygen level-dependent (BOLD) signals. We will model these using a fairly detailed dynamical model of the brain's neurovascular coupling.

\section{Simulating the BOLD Signal}

The simulation of fMRI BOLD signals appeals to the Balloon-Windkessel hemodynamic model of Friston et al. (2003). The Balloon-Windkessel model describes the coupling of perfusion to BOLD signal and is augmented with a dynamical model of the transduction of neural activity into perfusion changes. The model assumes that the BOLD signal is a static nonlinear function of normalized total deoxyhemoglobin voxel content, normalized venous volume, resting net oxygen extraction fraction, and resting blood volume fraction. In our model, the input to the hemodynamic model is neuronal activity summed over all neurons in both populations (excitatory and inhibitory populations) in any given area.

In brief, for the $i$ th region, neuronal activity $z_{i}$ causes an increase in a vasodilatory signal $s_{i}$ that is subject to autoregulatory feedback. The inflow $f_{i}$ responds in proportion to this signal with concomitant changes in blood volume $v_{i}$ and deoxyhemoglobin content $q_{i}$. The equations relating these biophysical variables are

$$
\begin{aligned}
\frac{\partial s_{i}(t)}{\partial t} & =z_{i}-k_{i} s_{i}-\gamma_{1}\left(f_{1}-1\right) \\
\frac{\partial f_{i}(t)}{\partial t} & =s_{1}
\end{aligned}
$$




$$
\begin{aligned}
& \frac{\tau_{i} \partial v_{i}(t)}{\partial t}=f_{i}-v_{1}^{\frac{1}{\alpha}} \\
& \frac{\tau_{i} \partial q_{i}(t)}{\partial t}=\frac{\left\{f_{i}\left[1-\left(1-\rho_{i}\right)\right]^{\frac{1}{f_{1}}}\right\}}{\rho_{i}}-\frac{v_{1}^{\frac{1}{\alpha}} q_{1}}{v_{1}},
\end{aligned}
$$

where $\rho$ is the resting oxygen extraction fraction. The BOLD signal is taken to be a static nonlinear function of volume and deoxyhemoglobin that comprises a volume-weighted sum of extravascular and intravascular signals:

$$
y_{i}=V_{0}\left(7 \rho_{i}\left(1-q_{i}\right)+2\left(1-\frac{q_{i}}{v_{i}}\right)+\left(2 \rho_{1}-0.2\right)\left(1-v_{1}\right)\right) \text {, }
$$

where $V_{0}=0.02$ is the resting blood volume fraction. The biophysical details of this model can be found in Friston et al. (2000).

We have now described a neuronal mass model of populations of neurons within each node of a distributed network. We have also discussed the coupling between these nodes at the macroscopic level in terms of empirically (anatomically) constrained extrinsic connectivity. Finally, we now have a model that maps from hidden neuronal states to observed hemodynamic responses in fMRI. In the next section, we will use this model to study some key emergent properties that illustrate the self-organization of brain dynamics described in the introduction.

\subsection{Autonomous Brain Dynamics}

Classical accounts of brain function (Hubel \& Wiesel, 1968; Barlow, 1990) emphasize the role of feedforward information processing in generating from the "ground up" sensory, cognitive, and motor representations that mediate behavior. Although feedforward sensory analysis can also be modulated by endogenous signals like familiarity, attention, and reward, a central tenet of this view is that any spontaneous or intrinsic activity reflects noise. But internal noise is crucial as it produces random (spike rate) fluctuations that can have a profound effect on the transmission of information. Such feedforward sensorimotor models have been successful in linking activity recorded from single neurons to perceptual decisions (Newsome, Britten, \& Movshon, 1989; Shadlen, Britten, Newsome, \& Movshon, 1996; Shadlen \& Newsome, 1996). However, a different class of models suggests that the brain is not a passive sensorimotor mapping, driven by sensory information, but that it actively generates and main- 
tains predictions (priors) about forthcoming sensory stimuli, cognitive states, and actions (Llinas, Ribary, Contreras, \& Pedroarena, 1998; Engel, Fries, \& Singer, 2001; Varela, Lachaux, Rodriguez, \& Martinerie, 2001; Friston, 2002). This class of models emphasizes the role of spontaneous ongoing activity in maintaining active and itinerant representations that are entrained rather than determined by sensory information. Accordingly, spontaneous ongoing activity should not be random (as often implied by its dismissal as mere "noise") but organized into structured spatiotemporal patterns that reflect the functional architecture of the brain, encode traces of previous behavior, or even predict future decisions. Hence, the study of whole-brain activity during stimulus-free (resting) conditions is a natural forum for examining these itinerant dynamics. The resulting endogenous, self-organized dynamics reflect the activity of the brain that can only arise from its intrinsic properties (neuroanatomic structure, local spontaneous brain areas dynamics, fluctuations, delays).

An increasing number of experimental studies have characterized the dynamics of spontaneous or ongoing activity with a variety of different methods including EEG (Creutzfeldt, Watanabe, \& Lux, 1966), optical imaging (Kenet et al., 2003), single unit (Engel et al., 2001), and fMRI (Biswal, Yetkin, Haughton, \& Hyde, 1995; Fox \& Raichle, 2007). In particular, fMRI measures local changes in magnetic susceptibility (BOLD signal) caused by variations in the capillary concentration of deoxyhemoglobin due to blood flow and blood volume increases in response to neuronal activation (see previous section). In the absence of stimuli, spontaneous fMRI signals are characterized by slow oscillations $(<0.1$ $\mathrm{Hz}$ ). It was noted more than a decade ago that spontaneous BOLD fluctuations are temporally correlated (or coherent) between brain regions with similar functional specialization (Biswal, DeYoe, \& Hyde, 1996); see (Fox \& Raichle, 2007) for review. The ensuing networks of correlated fluctuations are said to constitute resting-state networks. In what follows, we will look at these endogenous fluctuations using the large-scale model of neocortical dynamics introduced in the previous section (see also Ghosh, Rho, McIntosh, Kötter, \& Jirsa, 2008; Deco, Jirsa, McIntosh, Sporns, \& Kötter, 2009; Deco, Jirsa, \& McIntosh, 2011).

\subsubsection{Endogenous Activity and Multistability}

We now consider the dynamics of the realistic global attractor spiking network based on the neuroanatomic connectivity matrix of the macaque 
described earlier. We will see that by placing local dynamics on this macroscopic structure, we can explain many features of resting-state networks as evidenced by fMRI. Specifically, we find that resting-state networks can be accounted for by structured fluctuations around a trivial low-firing equilibrium state (fixed-point attractor). These fluctuations are induced by multistable attractors that correspond to high firing activity in particular brain areas.

We will first look at the stationary fixed points (attractor landscape) of the cortical network. To do this, we can reduce the spiking dynamics of the previous section to a set of mean field equations that describe the stationary states (see Brunel \& Wang, 2001; Deco, Jirsa, Robinson, Breakspear, \& Friston, 2008). This allows us to identify the fixed points as a function of the parameter $W$ that scales global coupling. Figure 1.3 shows the number of fixed points found in the system as a function of $W$. These fixed points were found by solving the mean-field equations from a thousand different initial conditions. Figure 1.3 also shows the average uncertainty (entropy) that a given attractor is occupied, when starting from random initial conditions.

This entropy characterizes the variability of cortical activity due to noise-driven transitions among multistable attractors. For very small

A

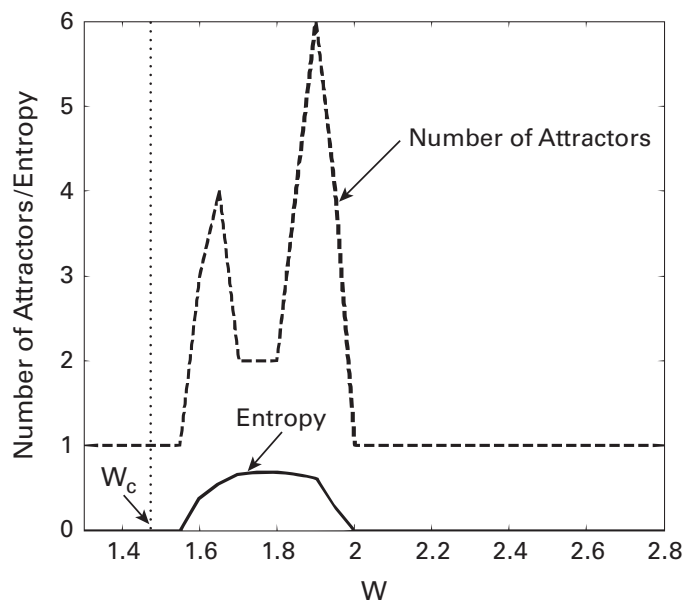

B

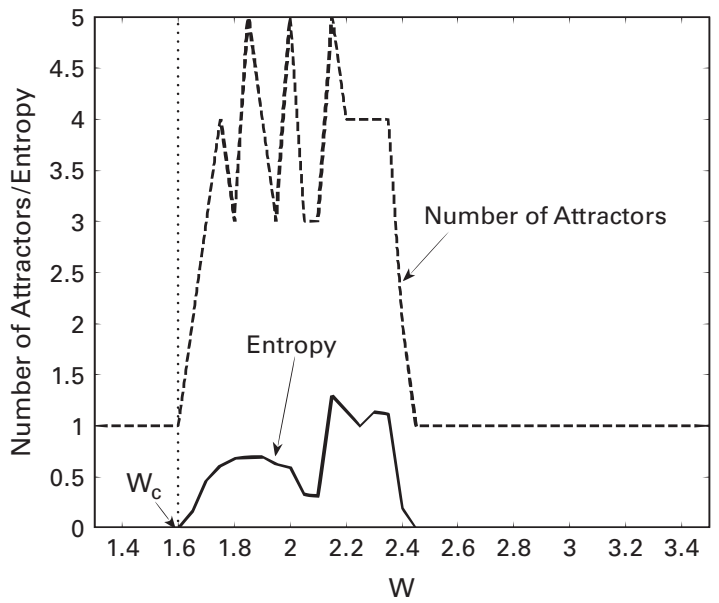

Figure 1.3

Mean field analyses of the attractor landscape of the cortical spiking network as a function of the global inter-areal coupling strength (left, monkey; right, human). The dashed line shows the number of stable attractors, whereas the continuous line shows the entropy of the attractors. 
values of $W$, only one attractor is stable, and therefore the entropy is zero. This fixed-point attractor corresponds to the trivial spontaneous ground state of the system, where all neurons are firing at a low level (excitatory neurons at $3 \mathrm{~Hz}$ and inhibitory neurons at $9 \mathrm{~Hz}$ ). For very large values of $W$, there is also only one stable attractor, and therefore the entropy is again zero. This attractor corresponds to the epileptiform case, where all excitatory neurons are highly activated in all brain areas. In intermediate regions of $W$, we find multistability corresponding to distinct foci of high firing activity in particular brain areas. This regime is reported by high entropy and contains many fixed-point attractors. It is this regime that characterizes real brain dynamics and, indeed, the dynamics of selforganized systems that maintain themselves near phase transitions or bifurcations. Similar phenomena are also seen using globally coupled maps based on oscillator models of neuronal dynamics. In these systems, increasingly global coupling takes the system from a completely desynchronized state, through a regime of multistability and itinerant chaos, to a quiescent regime of global synchronization.

To show that the brain may conform to similar principles of selforganization, we tested the hypothesis that simulated global dynamics in the multistable regime were a better predictor of observed fMRI dynamics obtained during resting state in humans: Using the spiking model, we calculated the neuronal activity in all brain areas and then simulated fMRI signals using the Balloon-Windkessel model (Friston et al., 2003). After simulated fMRI signals were downsampled to $2 \mathrm{~s}$ (Honey et al., 2007, they were treated in the same way as the empirical data: The global signal (average over all regions) was removed by linear regression (Fox et al., 2005, 2009). Finally, we computed the functional connectivity by calculating the correlation matrix of the BOLD activity among all brain areas. This was repeated for a range of global coupling parameters. To identify the region of the parameter $W$ where the model best reproduces the empirical functional connectivity, we computed the Pearson correlation between the empirical and the simulated functional connectivity matrices. Figure 1.4 shows that this is maximal for the critical values of global coupling found in figure 1.3. This result is important because it suggests the brain might also be exploiting multistability to maintain its rich repertoire of dynamics that are characteristic of endogenous neuronal activity. This repertoire depends upon an intermediate level of overall coupling among brain regions that is not too strong and is not too weak. We conclude with a brief discussion of the larger issues that this type of analysis is designed to address. 


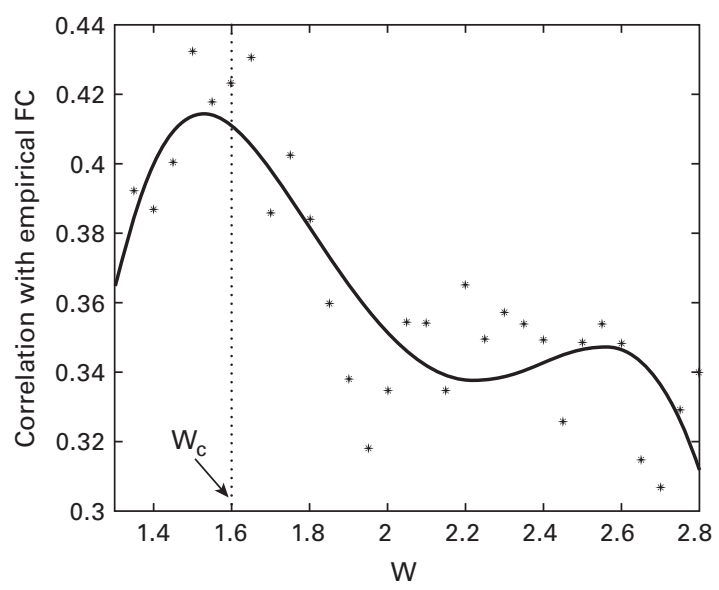

Figure 1.4

Fitting of empirical data in human subjects (as measured by the correlation between simulated and empirical functional connectivity) as a function of the global coupling parameter $W$. The best fit is achieved at the edge of the bifurcation.

\subsection{Conclusion}

These analyses above show that, even at rest, endogenous brain activity is self-organizing and highly structured. There are many questions about the genesis of autonomous dynamics and the structures that support them. Some of the more interesting come from computational anatomy and neuroscience. The emerging picture is that endogenous fluctuations are a consequence of dynamics on anatomic connectivity structures with particular scale-invariant and small-world characteristics (Achard, Salvador, Witcher, Suckling, \& Bullmore, 2006; Bassett \& Bullmore, 2009; Honey, Kötter, Breakspear, \& Sporns, 2007; Deco et al., 2009). These are well-studied and universal characteristics of complex systems and suggest that we may be able to understand the brain in terms of universal phenomena (Sporns, 2010). For example, Buice and Cowan (2009) model neocortical dynamics using field-theoretic methods (from nonequilibrium statistical processes) to describe both neural fluctuations and responses to stimuli. In their models, the density and extent of lateral cortical interactions induce a region of state space, in which the effects of fluctuations are negligible. However, as the generation and decay of neuronal activity comes into balance, there is a transition into a regime of critical fluctuations. These models suggest that the scaling laws, found in many measurements of neocortical activity, are consistent with the existence of phase transitions at a critical point. They also speak to larger 
questions about how the brain maintains itself near phase transitions (i.e., self-organized criticality and gain control; Abbott, Varela, Sen, \& Nelson, 1997; Kitzbichler, Smith, Christensen, \& Bullmore, 2009). This is an important issue, because systems near phase transitions show universal phenomena (Jirsa, Friedrich, Haken, \& Kelso, 1994; Jirsa \& Haken, 1996; Jirsa \& Kelso, 2000; Tschacher \& Haken, 2007; Tognoli \& Kelso, 2009). Although many people argue for criticality and power-law effects in large-scale cortical activity (e.g., Linkenkaer-Hansen, Nikouline, Palva, \& Ilmoniemi, 2001; Stam \& de Bruin, 2004; Freyer, Aquino, Robinson, Ritter, \& Breakspear, 2009; Kitzbichler et al., 2009), other people do not (Bedard, Kroger, \& Destexhe, 2006). It may be that slow (electrophysiologic) frequencies contain critical oscillations, whereas high-frequency coherent oscillations may reflect other dynamical processes. In short, endogenous fluctuations are an important example of dynamics and structure and may disclose fundamental principles of self-organization that underwrite the brain's remarkable capacity to support itinerant and adaptive dynamics.

\section{References}

Abbott, L. F., Varela, J. A., Sen, K., \& Nelson, S. B. (1997). Synaptic depression and cortical gain control. Science, 275(5297), 220-224.

Achard, S., Salvador, R., Whitcher, B., Suckling, J., \& Bullmore, E. (2006). A resilient, lowfrequency, small-world human brain functional network with highly connected association cortical hubs. Journal of Neuroscience, 26(1), 63-72.

Assisi, C. G., Jirsa, V. K., \& Kelso, J. A. S. (2005). Synchrony and clustering in heterogeneous networks with global coupling and parameter dispersion. Physical Review Letters, 94, 018106.

Barlow, H. (1990). What the brain tells the eye. Scientific American, 262, 90-95.

Bassett, D. S., \& Bullmore, E. T. (2009). Human brain networks in health and disease. Current Opinion in Neurology, 22(4), 340-347.

Bedard, C., Kroger, H., \& Destexhe, A. (2006). Model of low-pass filtering of local field potentials in brain tissue. Physical Review E: Statistical, Nonlinear, and Soft Matter Physics, 73(5 Pt 1), 051911.

Biswal, B., DeYoe, A. E., \& Hyde, J. S. (1996). Reduction of physiological fluctuations in fMRI using digital filters. Magnetic Resonance in Medicine, 35, 107-113.

Biswal, B., Yetkin, F., Haughton, V., \& Hyde, J. (1995). Functional connectivity in the motor cortex of resting human brain using echo-planar MRI. Magnetic Resonance in Medicine, $34,537-541$.

Brunel, N., \& Wang, X. J. (2001). Effects of neuromodulation in a cortical network model of object working memory dominated by recurrent inhibition. Journal of Computational Neuroscience, 11, 63-85.

Buice, M. A., \& Cowan, J. D. (2009). Statistical mechanics of the neocortex. Progress in Biophysics and Molecular Biology, 99(2-3), 53-86.

Cabeza, R., \& Nyberg, L. (2000). Imaging cognition II: empirical review of 275 PET and fMRI studies. Journal of Cognitive Neuroscience, 12, 1-47. 
Cordes, D., Haughton, V., Arfanakis, K., Wendt, G., Turski, W., Moritz, C., et al. (2000). Mapping functionally related regions of brain with functional connectivity MR imaging. AJNR. American Journal of Neuroradiology, 21, 1636-1644.

Creutzfeldt, O., Watanabe, S., \& Lux, H. (1966). Relations between EEG phenomena and potentials of single cortical cells. Electroencephalography and Clinical Neurophysiology, 20, 19-37.

Deco, G., Jirsa, V., \& McIntosh, A. R. (2011). Emerging concepts for the dynamical organization of resting-state activity in the brain. Nature Reviews. Neuroscience, 12, 43-56.

Deco, G., Jirsa, V., McIntosh, A. R., Sporns, O., \& Kötter, R. (2009). Key role of coupling, delay, and noise in resting brain fluctuations. Proceedings of the National Academy of Sciences of the United States of America, 106, 10302-10307.

Deco, G., Jirsa, V., Robinson, P., Breakspear M., and Friston, K. (2008). The dynamic brain: From spiking neurons to neural masses and cortical fields. PLoS Computational Biology 4 (8), e1000092.

Engel, A., Fries, P., \& Singer, W. (2001). Dynamic predictions: oscillations and synchrony in top-down processing. Nature Reviews. Neuroscience, 2, 704-716.

Feinberg, T. E., \& Farah, M. J. (1997). The development of modern behavioral neurology and neuropsychology. In T. E. Feinberg \& M. J. Farah (Eds.), Behavioral Neurology and Neuropsychology. New York: McGraw Hill.

Flourens, M. J. P. (1824). Recherches expérimentales sur les propriétés et les fonctions du système nerveux, dans les animaux vertébrés (1st ed., Vol. 26, p. 20). Paris: Chez Crevot.

Fox, M. D., Snyder, A. Z., Vincent, J. L., Corbetta, M., Van Essen, D. C., et al. (2005). The human brain is intrinsically organized into dynamic, anticorrelated functional networks. Proceedings of the National Academy of Sciences USA, 102, 9673-9678.

Fox, M., \& Raichle, M. (2007). Spontaneous fluctuations in brain activity observed with functional magnetic resonance imaging. Nature Reviews. Neuroscience, 8, 700-711.

Fox, M. D., Zhang, D., Snyder, A. Z., \& Raichle, M. E. (2009).The global signal and observed anticorrelated resting state brain networks. Journal of Neurophysiology, 101, 3270-3283.

Freyer, F., Aquino, K., Robinson, P. A., Ritter, P., \& Breakspear, M. (2009). Non-Gaussian statistics in temporal fluctuations of spontaneous cortical activity. Journal of Neuroscience, $29,8512-8524$.

Friston, K. J., Mechelli, A., Turner, R., \& Price, C. J. (2000). Nonlinear responses in fMRI: The Balloon model, Volterra kernels, and other hemodynamics. Neuroimage, 12, 466-477.

Friston, K. (2002). Beyond phrenology: what can neuroimaging tell us about distributed circuitry? Annual Review of Neuroscience, 25, 221-250.

Friston, K., Harrison, L., \& Penny, W. (2003). Dynamic causal modelling. NeuroImage, 19, 1273-1302.

Gall, F. J., \& Spurzheim, J. G. (1967). Recherches sur le Systeme Nerveux. Amsterdam: Bonset. Originally published in 1809.

Ghosh, A., Rho, Y., McIntosh, A. R., Kötter, R., \& Jirsa, V. K. (2008). Noise during rest enables the exploration of the brain's dynamic repertoire. PLoS Computational Biology, 4, e1000196.

Hagmann, P., Cammoun, L., Gigandet, X., Meuli, R., Honey, C. J., Wedeen, V. J., \& Sporns, O. (2008). Mapping the structural core of human cerebral cortex. PLoS Biology, 6, e159.

Honey, C. J., Kötter, R., Breakspear, M., \& Sporns, O. (2007). Network structure of cerebral cortex shapes functional connectivity on multiple time scales. Proceedings of the National Academy of Sciences of the United States of America, 104, 10240-10245.

Hubel, D. H., \& Wiesel, T. N. (1968). Receptive fields and functional architecture of monkey striate cortex. Journal of Physiology, 195, 215-243.

Jirsa, V. K. (2007). Dispersion and time-delay effects in synchronized spike-burst networks. Cognitive Neurodynamics, 2(1), 29-38. 
Jirsa, V. K., \& Haken, H. (1996). Field theory of electromagnetic brain activity. Physical Review Letters, 77, 960-963.

Jirsa, V. K., \& Kelso, J. A. (2000). Spatiotemporal pattern formation in neural systems with heterogeneous connection topologies. Physical Review E: Statistical Physics, Plasmas, Fluids, and Related Interdisciplinary Topics, 62 (6 Pt B), 8462-8465.

Jirsa, V. K., Friedrich, R., Haken, H., \& Kelso, J. A. (1994). A theoretical model of phase transitions in the human brain. Biological Cybernetics, 71(1), 27-35.

Jirsa, V. K., \& Stefanescu, R. A. (2010). Neural population modes capture biologically realistic large scale network dynamics. Bulletin of Mathematical Biology, 73, 325-343.

Jirsa, V. K., Sporns, O., Breakspear, M., Deco, G., \& McIntosh, A. R. (2010). Towards the virtual brain: network modeling of the intact and the damaged brain. Archives Italiennes de Biologie, 148, 189-205.

Kenet, T., Bibitchkov, D., Tsodyks, M., Grinvald, A., \& Arieli, A. (2003). Spontaneously emerging cortical representations of visual attributes. Nature, 425, 954-956.

Kitzbichler, M. G., Smith, M. L., Christensen, S. R., \& Bullmore, E. (2009). Broadband criticality of human brain network synchronization. PLoS Computational Biology, 5(3), e1000314.

Knock, S. A., McIntosh, A. R., Sporns, O., Kötter, R., Hagmann, P., \& Jirsa, V. K. (2009). The effects of physiologically plausible connectivity structure on local and global dynamics in large scale brain models. Journal of Neuroscience Methods, 183, 86-94.

Kötter, R., \& Wanke, E. (2005). Mapping brains without coordinates. Philosophical Transactions of the Royal Society of London. Series B, 360, 751-766.

Linkenkaer-Hansen, K., Nikouline, V. V., Palva, J. M., \& Ilmoniemi, R. J. (2001). Long-range temporal correlations and scaling behavior in human brain oscillations. Journal of Neuroscience, 21, 1370-1377.

Llinas, R., Ribary, U., Contreras, D., \& Pedroarena, C. (1998). The neuronal basis for consciousness. Philosophical Transactions of the Royal Society of London. Series B, Biological Sciences, 353, 1841-1849.

McIntosh, R., \& Gonzalez-Lima, F. (1994). Structural equation modeling and its application to network analysis in functional brain imaging. Human Brain Mapping, 2, 2-22.

Newsome, W. T., Britten, K. H., \& Movshon, J. A. (1989). Neuronal correlates of a perceptual decision. Nature, 341, 52-54.

Shadlen, M., \& Newsome, W. (1996). Motion perception: seeing and deciding. Proceedings of the National Academy of Sciences of the United States of America, 93, 628-633.

Shadlen, M., Britten, K., Newsome, W., \& Movshon, J. A. (1996). Computational analysis of the relationship between neuronal and behavioral responses to visual motion. Journal of Neuroscience, 16, 1486-1510.

Sporns, O. (2010). Networks of the Brain. Cambridge, MA: MIT Press.

Stam, C. J., \& de Bruin, E. A. (2004). Scale-free dynamics of global functional connectivity in the human brain. Human Brain Mapping, 22, 97-109.

Stefanescu, R., \& Jirsa, V. K. (2008). A low dimensional description of globally coupled heterogeneous neural networks of excitatory and inhibitory neurons. PLoS Computational Biology, 4(11), e1000219.

Stefanescu, R., \& Jirsa, V. K. (2011). Reduced neural population dynamics with synaptic coupling. PRE.

Tognoli, E., \& Kelso, J. A. (2009). Brain coordination dynamics: true and false faces of phase synchrony and metastability. Progress in Neurobiology, 87(1), 31-40.

Tschacher, W., \& Haken, H. (2007). Intentionality in non-equilibrium systems? The functional aspects of self-organised pattern formation. New Ideas in Psychology, 25, 1-15.

Varela, F., Lachaux, J., Rodriguez, E., \& Martinerie, J. (2001). The brainweb: phase synchronization and large-scale integration. Nature Reviews. Neuroscience, 2, 229-239. 